\title{
A New Eighth Order Runge-Kutta Family Method
}

\author{
Séka Hippolyte ${ }^{1}$, Assui Kouassi Richard ${ }^{2}$ \\ ${ }^{1}$ Département de Mathématiques et Informatique, Institut National Polytechnique Houphouet-Boigny, Yamoussoukro, \\ Côte d'Ivoire \\ ${ }^{2}$ Département de Mathématiques et Informatique, Institut National Polytechnique Houphouet-Boigny, Yamoussoukro, \\ Côte d'Ivoire \\ Correspondence: Séka Hippolyte, Institut National Polytechnique Houphouet-Boigny, Yamoussoukro. Bp 1093 Yamous- \\ soukro, Côte d'Ivoire. E-mail: hippolyte.seka@inphb.ci
}

Received: March 2, 2019 Accepted: March 20, 2019 Online Published: March 25, 2019

doi:10.5539/jmr.v11n2p190 URL: https://doi.org/10.5539/jmr.v11n2p190

\begin{abstract}
In this article, a new family of Runge-Kutta methods of $8^{\text {th }}$ order for solving ordinary differential equations is discovered and depends on the parameters $b_{8}$ and $a_{10,5}$. For $b_{8}=49 / 180$ and $a_{10,5}=1 / 9$, we find the Cooper-Verner method [1]. We show that the stability region depends only on coefficient $a_{10,5}$. We compare the stability regions according to the values of $a_{10,5}$ with respect to the stability region of Cooper-Verner.
\end{abstract}

Keywords: Runge-Kutta, ordinary differential equations, Cooper-Verner, region of stability

\section{Introduction}

Since the time of Newton, one of the main problems of mathematicians is the resolution of various differential equations. Practically, the immense quantity of these equations were not resolvable in the analytical aspect. This has led to the development of numerical methods for their resolution. The method of Runge-Kutta, named RK is used to find a good numerical result. Currently, a large number of high-order RK methods are known (5..10) [1, 2, 4, 8, 9, 10]. However, they are not all found. For example, methods of $8^{\text {th }}$ order depending of several parameters are not presented in the literature. The field of stability has not been fully investigated. In this article we discover new family of order 8 depending on several parameters. This family summarizes the Cooper-Verner method [1]. It depends on the coefficients $b_{8}$ and $a_{10,5}$. For $b_{8}=49 / 180$ and $a_{10,5}=1 / 9$, we find the Cooper-Verner method [1]. Subsequently, we show that the region of stability of this family depends only on the coefficient $a_{10,5}$ and not on $b_{8}$. The study will consist in comparing the regions of stability according to the values of $a_{10,5}$ with respect to the stability region from Cooper-Verner.

The study will be led by respecting the following plan: in section 2 Presentation of the new family RK8 method, section 3 The stability region, section 4 Comparison of some stability regions, section 5 Conclusion.

\section{Presentation of the New Family RK8 Method}

Consider a general form of the first-order ODE given below:

$$
y^{\prime}=f(x, y(x))
$$

with the initial condition $y\left(x_{0}\right)=y_{0}$ for the interval $x_{0} \leq x \leq x_{n}$. Here, $x$ is the independent variable, $y$ is the dependent variable, $n$ is the number of point values, and $f$ is the function of the derivation. The goal is to determine the unknown function $y(x)$ whose derivative satisfies (1) and the corresponding initial values. In doing so, let us discretize the interval $x_{0} \leq x \leq x_{n}$ to be

$$
x_{0}, x_{1}=x_{0}+h, x_{2}=x_{0}+2 h, \ldots, x_{n}=x_{0}+n h,
$$

where $h$ is the fixed step size. With the initial condition $y\left(x_{0}\right)=y_{0}$, the unknown function $y_{1}, y_{2}, y_{3}, \cdots, y_{n}$ can be solved by using the RK8 method.

The family of $8^{\text {th }}$ order method is thus obtained by the resolution of the 200 equations with 11 stages (see Appendix A) on Maple. However, the 200 equations are obtained from the successive derivatives of the exact solution up to 8 (see Appendix B).

Lets consider the Butcher tableau of 8 order 11 steps RK method(see Fig. 1): 


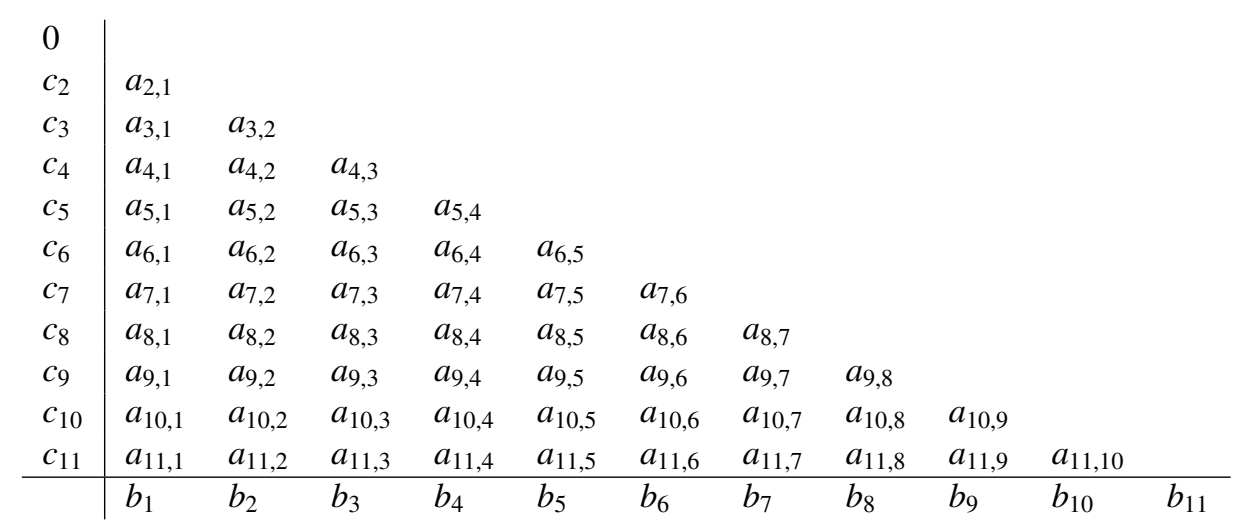

Figure 1. Butcher tableau of RK8 family

with free parameters $b_{8}$ and $a_{10,5}$.

Some of these coefficients have fixed values, not depending on $b_{8}$ and $a_{10,5}$, these coefficients are:

$$
\begin{gathered}
b_{1}=\frac{1}{20} ; b_{2}=0 ; b_{3}=0 ; b_{4}=0 ; b_{5}=0 ; b_{6}=0 ; b_{9}=\frac{16}{45} ; b_{10}=\frac{49}{180} ; b_{11}=\frac{1}{20} ; \\
c_{2}=\frac{1}{2} ; c_{3}=\frac{1}{2} ; c_{4}=\frac{7+\sqrt{21}}{14} ; c_{5}=\frac{7+\sqrt{21}}{14} ; c_{6}=\frac{1}{2} ; \\
c_{7}=\frac{7-\sqrt{21}}{14} ; c_{8}=\frac{7-\sqrt{21}}{14} ; c_{9}=\frac{1}{2} ; c_{10}=\frac{7+\sqrt{21}}{14} ; c_{11}=1 \\
a_{2,1}=\frac{1}{2} ; \\
a_{3,1}=\frac{1}{4} ; a_{3,2}=\frac{1}{4} ; \\
a_{4,1}=\frac{1}{7} ; a_{4,2}=\frac{-7-3 \sqrt{21}}{98} ; a_{4,3}=\frac{21+5 \sqrt{21}}{49} ; \\
a_{5,1}=\frac{11+\sqrt{21}}{84} ; a_{5,2}=0 ; a_{5,3}=\frac{4 \sqrt{21}}{63}+\frac{2}{7} ; a_{5,4}=\frac{21-\sqrt{21}}{252} ; \\
48
\end{gathered} ; \begin{gathered}
a_{6,2}=0 ; a_{6,3}=\frac{9+\sqrt{21}}{36} ; a_{6,4}=\frac{-231+14 \sqrt{21}}{360} ; a_{6,5}=\frac{63-7 \sqrt{21}}{80} ; \\
a_{7,1}=\frac{10-\sqrt{21}}{42} ; a_{7,2}=0 ; \\
a_{9,1}=\frac{1}{32} ; a_{9,2}=0 ; \\
a_{10,1}=\frac{1}{14} ; a_{10,2}=0 ; a_{10,9}=\frac{4 \sqrt{21}}{35}+\frac{132}{245} ; \\
a_{11,1}=0 ; a_{11,2}=0 ; a_{11,9}=\frac{28-28 \sqrt{21}}{45} ; a_{11,10}=\frac{49-7 \sqrt{21}}{18} ;
\end{gathered}
$$

And the others are expressed in terms of $b_{8}$ and $a_{10,5}$ :

$$
\begin{gathered}
b_{7}=-b_{8}+\frac{49}{180} ; \\
a_{7,3}=-(24 / 35) a_{10,5}-136 / 105-(12 / 245) a_{10,5} \sqrt{21}+(656 / 2205) \sqrt{21}
\end{gathered}
$$




$$
\begin{aligned}
& a_{7,4}=7-(3 / 10) a_{10,5} \sqrt{21}-(71 / 45) \sqrt{21}+(3 / 10) a_{10,5} ; \\
& a_{7,5}=-(3 / 10) a_{10,5}+(3 / 10) a_{10,5} \sqrt{21}-43 / 6+(169 / 105) \sqrt{21} ; \\
& a_{7,6}=-(277 / 735) \sqrt{21}+181 / 105+(12 / 245) a_{10,5} \sqrt{21}+(24 / 35) a_{10,5} ; \\
& a_{8,1}=-\frac{180 b_{8} \sqrt{21}-49 \sqrt{21}-1800 b_{8}+343}{7560 b_{8}} ; a_{8,2}=0 ; \\
& a_{8,5}=-\frac{441 a_{10,5} \sqrt{21}-3240 a_{7,5} b_{8}-28 \sqrt{21}+882 a_{7,5}-2205 a_{10,5}+147}{3240 b_{8}} ; \\
& a_{8,6}=\frac{72 a_{10,5} \sqrt{21}+1620 a_{7,6} b_{8}-29 \sqrt{21}-441 a_{7,6}-252 a_{10,5}+119}{1620 b_{8}} ; \\
& a_{8,3}=-\frac{900 b_{8} \sqrt{21}+11340 a_{7,2} b_{8}+11340 a_{8,6} b_{8}-98 \sqrt{21}-3087 a_{7,2}-4860 b_{8}+686}{11340 b_{8}} ; \\
& a_{8,7}=\frac{49}{1620 b_{8}} ; \\
& a_{8,4}=\frac{\left(c_{8}^{2} / 2\right)-a_{8,2} c_{2}-a_{8,3} c_{3}-a_{8,5} c_{5}-a_{8,6} c_{6}-a_{8,7} c_{7}}{c_{4}} ; \\
& a_{9,3}=(1 / 8) a_{10,5} \sqrt{21}-(1 / 8) a_{10,5}-(1 / 72) \sqrt{21}+1 / 72 ; \\
& a_{9,4}=-49 / 288-(7 / 32) a_{10,5} \sqrt{21}+(7 / 288) \sqrt{21}+(49 / 32) a_{10,5} \text {; } \\
& a_{9,5}=(7 / 32) a_{10,5} \sqrt{21}-(35 / 576) \sqrt{21}-(49 / 32) a_{10,5}+21 / 64 ; \\
& a_{9,6}=-(1 / 8) a_{10,5} \sqrt{21}+(1 / 8) a_{10,5}+(1 / 72) \sqrt{21}+5 / 36 ; \\
& a_{9,7}=91 / 576+(7 / 192) \sqrt{21}-(585 / 1568) b_{8} \sqrt{21}-(405 / 224) b_{8} ; \\
& a_{9,8}=(585 / 1568) b_{8} \sqrt{21}+(405 / 224) b_{8} ; \\
& a_{10,3}=-(6 / 49) a_{10,5} \sqrt{21}-(2 / 7) a_{10,5}+(2 / 147) \sqrt{21}+2 / 63 \text {; } \\
& a_{10,4}=1 / 9-a_{10,5} \text {; } \\
& a_{10,6}=(2 / 7) a_{10,5}-803 / 2205+(6 / 49) a_{10,5} \sqrt{21}-(59 / 735) \sqrt{21} \text {; } \\
& a_{10,7}=1 / 9+(1 / 42) \sqrt{21}+(2295 / 686) b_{8}+(495 / 686) b_{8} \sqrt{21} \text {; } \\
& a_{10,8}=-(2295 / 686) b_{8}-(495 / 686) b_{8} \sqrt{21} ; \\
& a_{11,3}=(2 / 3) a_{10,5} \sqrt{21}-(2 / 3) a_{10,5}-(2 / 27) \sqrt{21}+2 / 27 ; \\
& a_{11,4}=-(7 / 6) a_{10,5} \sqrt{21}+(7 / 54) \sqrt{21}+(49 / 6) a_{10,5}-49 / 54 \text {; } \\
& a_{11,5}=(7 / 27) \sqrt{21}-77 / 54-(49 / 6) a_{10,5}+(7 / 6) a_{10,5} \sqrt{21} ; \\
& a_{11,6}=(2 / 3) a_{10,5}-64 / 135-(2 / 3) a_{10,5} \sqrt{21}+(94 / 135) \sqrt{21} \text {; } \\
& a_{11,7}=7 / 18-(265 / 98) b_{8} \sqrt{21}-(215 / 14) b_{8} ; \\
& a_{11,8}=(265 / 98) b_{8} \sqrt{21}+(215 / 14) b_{8} ;
\end{aligned}
$$

The numerical solution is given by the formula

$$
y_{i+1}=y_{i}+h\left(\sum_{s=1}^{11} b_{s} k_{s}\right)
$$

with

$$
k_{s}=f\left(x_{i}+c_{s} h, y_{i}+h \sum_{j=1}^{s-1} a_{s, j} k_{j}\right), \quad x_{i+1}=x_{i}+h
$$


We can notice that if $b_{8}=49 / 180$ and $a_{10,5}=1 / 9$, then we find the method of Cooper-Verner [1].

\section{The Stability Regions}

The concept of stability is based on the discrete solution. It gives back account of the actual behavior of the approximate solution for a given practical value, therefore non-zero, of the $h$ step. In real calculation, the errors accumulate. This is particularly evident in the process of solving a differential equation where one progresses step by step from an initial value. There are various stability conditions. First of all the numerical solution must remain limited. This requirement of minimum stability may be insufficient in practice, the bound obtained being often an exponential of the duration which therefore grows infinitely when it increases. We then introduce more stability criteria, demanding that the digital solution reproduce the behavior physical of the exact solution. The concept of absolute stability, in its simplest form, is based on the analysis of the behavior, according to the values of the step $h$, of the numerical solutions of the equation model $[1,3,5,6]$ :

$$
u^{\prime}(t)=\lambda u(t)
$$

Using (44) and (45), we obtain:

$$
\begin{gathered}
k_{1}=\lambda y_{i} ; \\
\text { for } s>1, k_{s}=\lambda\left(y_{i}+h \sum_{j=1}^{s-1} a_{s, j} k_{j}\right)
\end{gathered}
$$

Which give:

Let's put $z=h \lambda$. We obtain by Maple:

$$
y_{i+1}=\zeta(h \lambda) y_{i}
$$

$$
\begin{gathered}
\zeta(z)=1-z+(1 / 2) z^{2}-(1 / 6) z^{3}+(1 / 24) z^{4}-(1 / 120) z^{5}+(1 / 720) z^{6}-(1 / 5040) z^{7}+(1 / 40320) z^{8}+ \\
(797 / 50803200) z^{9}+(499 / 152409600) \sqrt{21} z^{9}-(1 / 25200) z^{9} a_{10,5}-(37 / 4233600) \sqrt{21} z^{9} a_{10,5}+ \\
(1 / 470400) z^{10}+(1 / 2083725) \sqrt{21} z^{10}-(31 / 940800) z^{10} a_{10,5}-(61 / 8467200) \sqrt{21} z^{10} a_{10,5} \\
(13 / 4267468800) \sqrt{21} z^{11}-(11 / 1612800) z^{11} a_{10,5}-(353 / 237081600) \sqrt{21} z^{11} a_{10,5}+(1 / 29030400) z^{11}
\end{gathered}
$$

The absolute stability region is the set

$$
\{z \in \mathbb{C}|| \zeta(z) \mid \leq 1\}
$$

\section{Comparison of Some Stability Regions}

The stability region depends on coefficient $a_{10,5}$. We obtain by Maple different regions according to values of $a_{10,5}$.

For $a_{10,5}=1 / 9$, we obtain the stability region of the Cooper-Verner method (see Fig. 2). From the point of view of the values of $x$, if we choose $a_{10,5}=\frac{1}{10}$ (see Fig. 3) or $a_{10,5}=\frac{1}{10.9}$ ( see Fig. 4) or $a_{10,5}=\frac{1}{11.9}$ ( see Fig. 5), then we see that the stability regions are bigger than the region of Cooper-Verner.

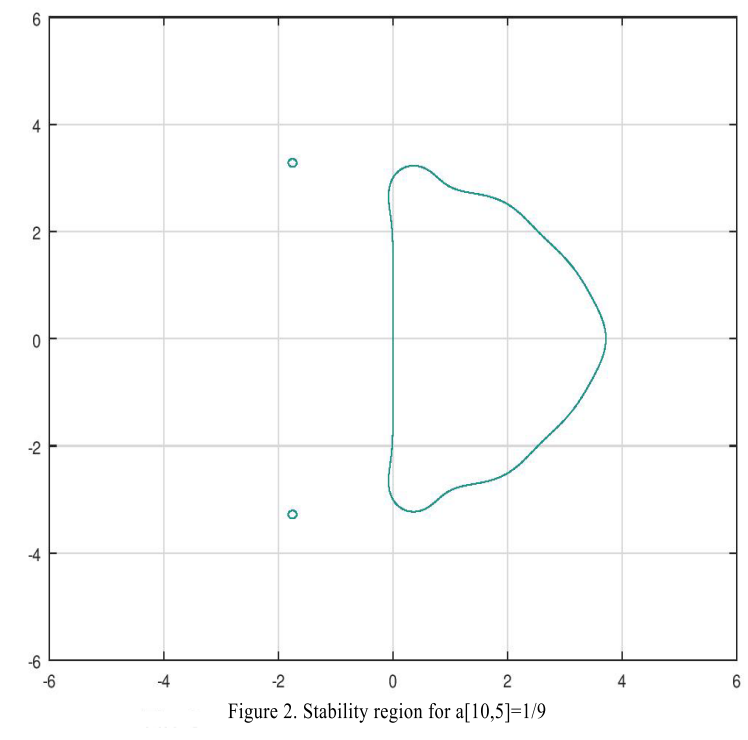

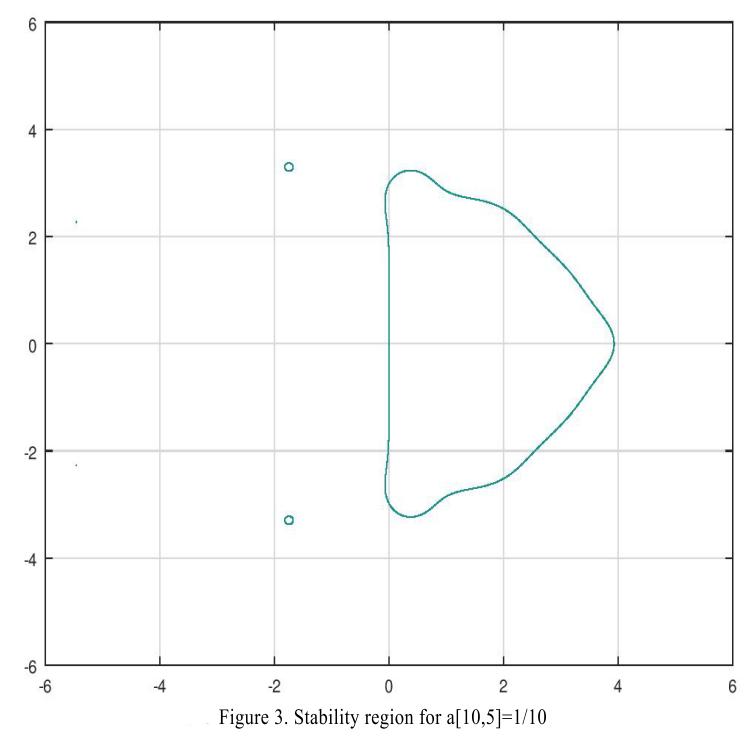



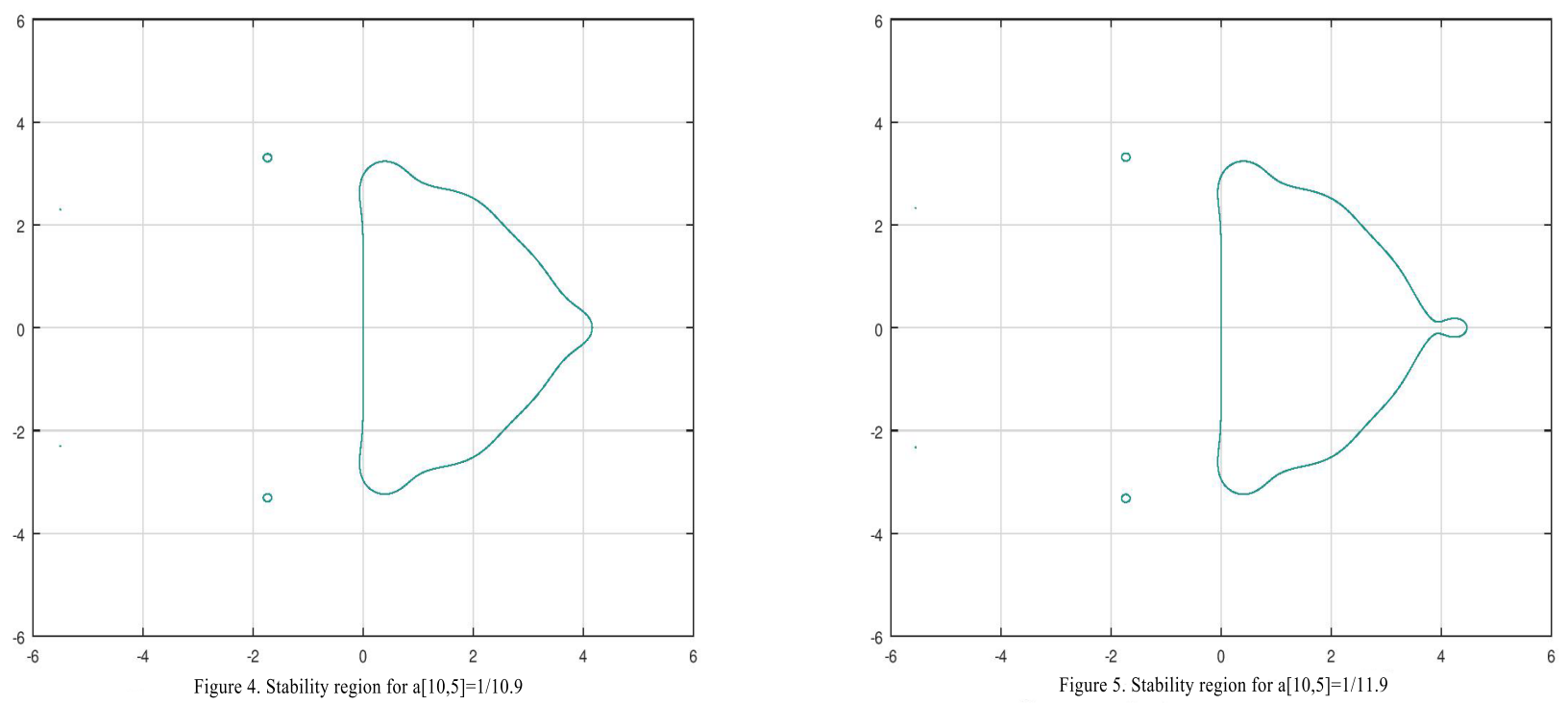

Let's see some stability region of $a_{10,5}=\{1,10,100,1000\}$. (see Fig. 6, Fig. 7, Fig. 8, Fig. 9). We see that the more $a_{10,5}>1$, the more the stability region becomes smaller. We can notice that all stability regions are smaller than of the Cooper-Verner one.
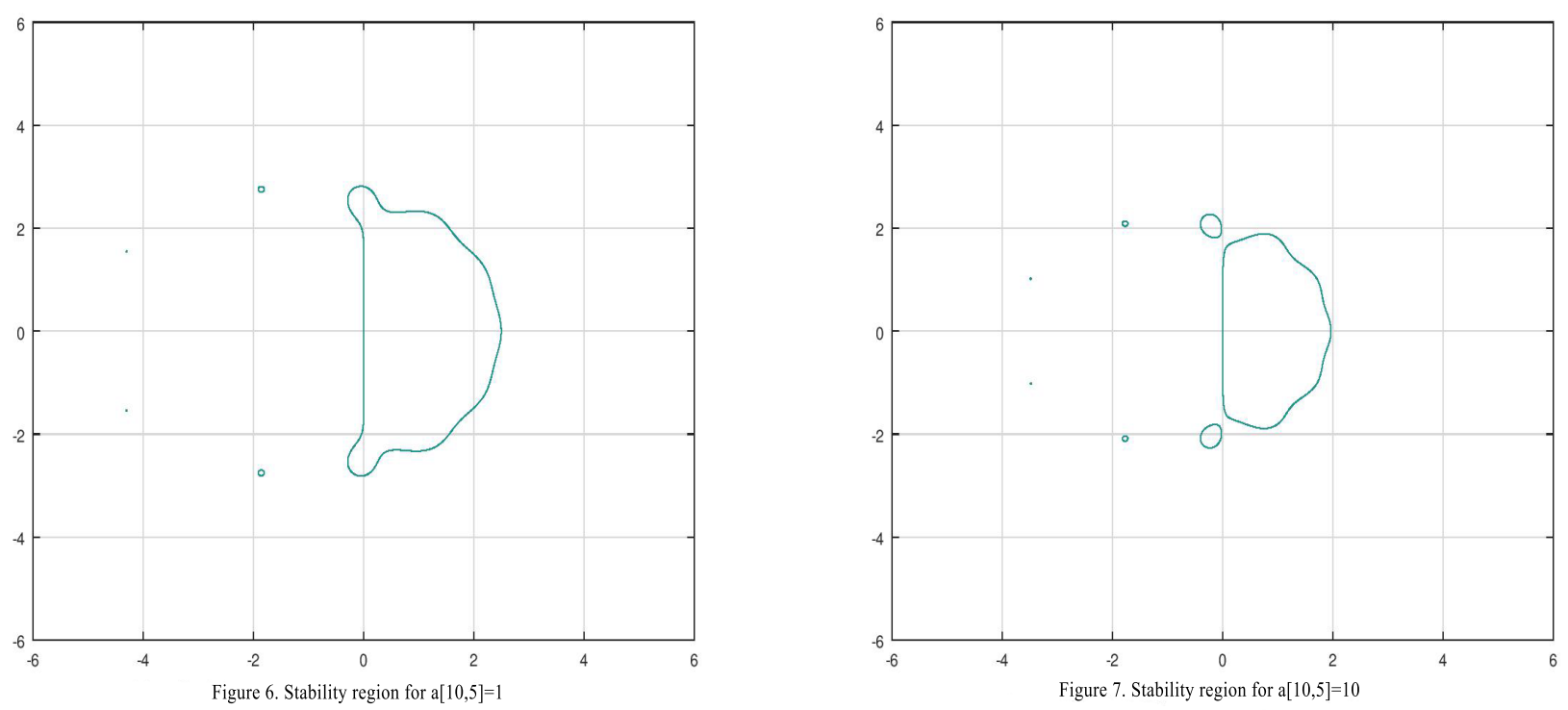

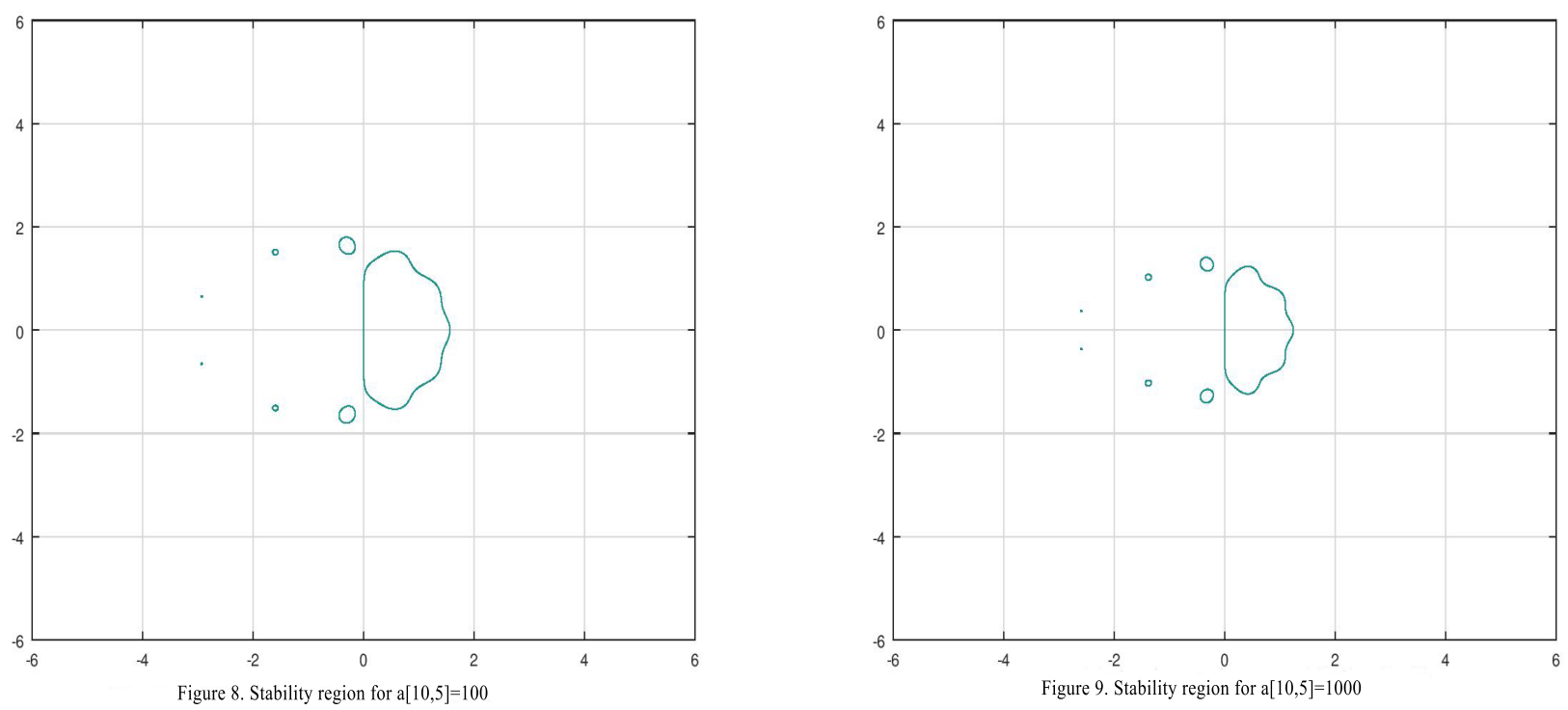

Let's see some stability region of $a_{10,5}=\{0,-1,-10,-100\}$. (see Fig. 10, Fig. 11, Fig. 12, Fig. 13). We can see that the more $a_{10,5}<0$, the more the stability region is smaller. We also notice that all the stability regions are smaller than that of the Cooper-Verner one.
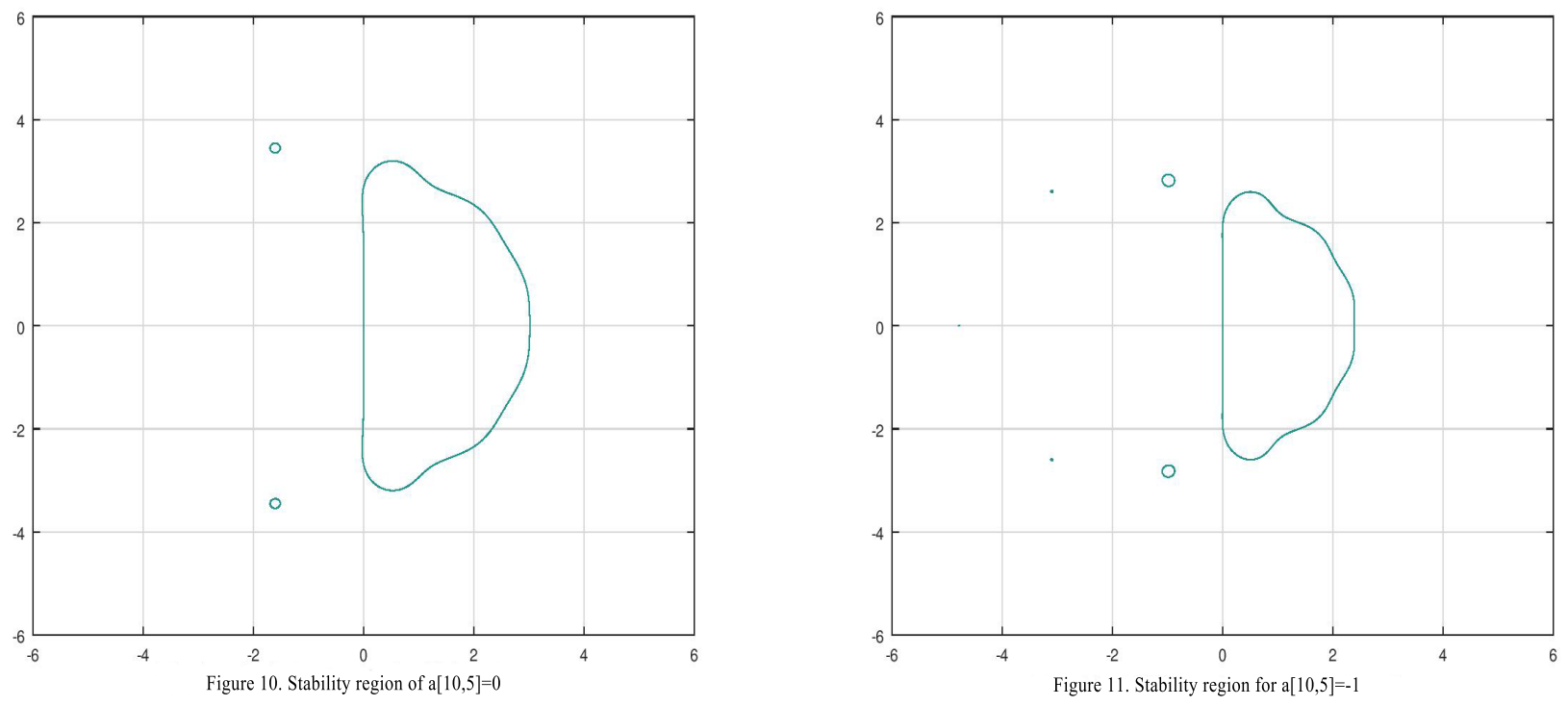

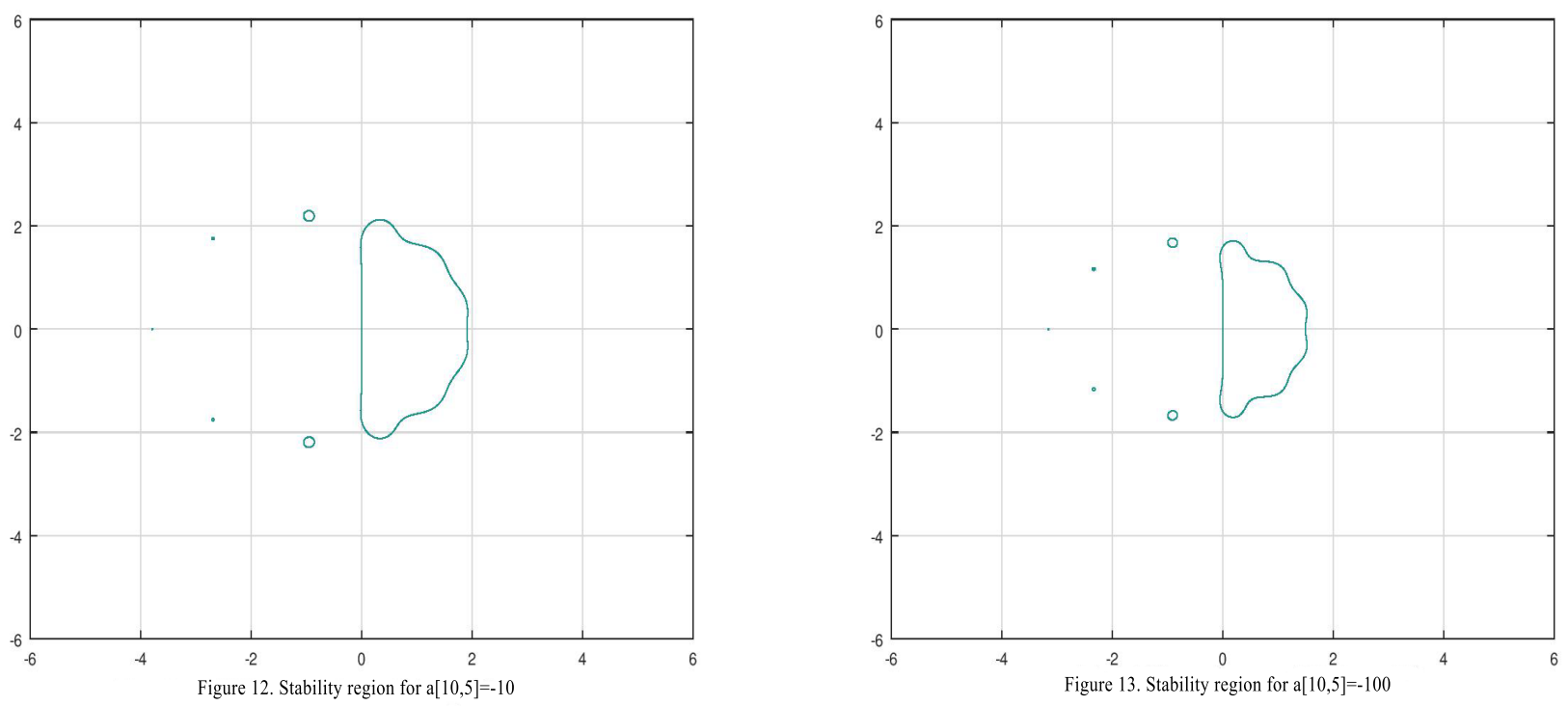

\section{Conclusion}

A new family of Runge-Kutta method $8^{\text {th }}$ order is discovered. This family depends one the parameters $a_{10,5}$ and $b_{8}$. For $a_{10,5}=1 / 9$ and $b_{8}=49 / 180$, we find the method of Cooper-Verner. The stability region depends on the value of $a_{10,5}$ but not of the $b_{8}$ coefficient. If we want to obtain a region of stability greater than that of the Cooper-Verner method, then it is not better to choose negative values of $a_{10,5}$ or values greater than 1 . You have to choose wisely $a_{10,5}$ between 0 and 1 .

\section{Acknowledgements}

We would like to express our deepest appreciation and gratitude to Professor Sergey Khashin of Ivanovo State University who provided us the possibility to coordinate and complete this article.

\section{References}

Butcher, J-C. (2008). Numerical Methods for Ordinary Differential Equations (2nd ed.): Wiley. https://doi.org/10.1002/9780470753767

Calvo, M., Montijano, J. I., \& Randez, L. (1990). A new embedded pair of Runge-Kutta formulas of orders 5 and 6. Computers \& Mathematics with Applications, 20(1), 15-24. https://doi.org/10.1016/0898-1221(90)90064-Q

Cassity, C. R. (1969). The complete solutions of the Fifth-Order Runge-Kutta Equations. SIAM Journal on Numerical Analysis . 3(4), 598-606. https://doi.org/10.1137/0706038

Feagin, T. (2007). A tenth-order Runge-Kutta method with error estimate[PDF notes]. Retrieved from https://sce.uhcl.edu/feagin/courses/rk10.pdf

Feagin, T. (2013). High-Order Explicit Runge-Kutta Methods[Web slides]. Retrieved from http://sce.uhcl.edu/rungekutta

Hairer, E., Norsett, S. P., \& Wanner, G. (1993). Solving ordinary differential equations I. Nonstiff Problems. https://doi.org/10.1007/978-3-540-78862-1

Jackiewicz, Z. (2009). General Linear Methods for Ordinary Differential Equations. https://doi.org/10.1002/9780470522165

Khashin, S. I. (2013). List of some known Runge-Kutta methods family(Preliminary version). Retrieved from http://math.ivanovo.ac.ru/dalgebra/Khashin/rk/sh_rk.html

Kashin, S. I. (2014). Estimating the error in classical Runge-Kutta methods. Computational Mathematics and Mathematical Physics, 54(5), 767-774. https://doi.org/10.1134/S0965542514050145

Liu, M. Z., Song, M. H., \& Yang, Z. W. (2004). Stability of Runge-Kutta methods in the numerical solution of equation $u^{\prime}(t)=a u(t)+a_{0} u([t])$. Journal of Computational and Applied Mathematics, 166(2), 361-370. https://doi.org/10.1016/j.cam.2003.04.002

Stephan, H. (2002). Stability regions of Runge-Kutta methods [PDF notes]. Retrieved from https://www.win.tue.nl/casa/meetings/seminar/previous/_abstract020220_files/talk.pdf 
Velagala, \& Sindhuja. (2014). Stability analysis of the 4th order Runge-Kutta method in application to colloidal particle interactions. (Master's thesis, University of Illinois, Urbana-Champaign, USA). Retrieved from http://hdl.handle.net/2142/72750

\section{Appendix A}

\section{The 200 equations of order 8 with $s$ stages}

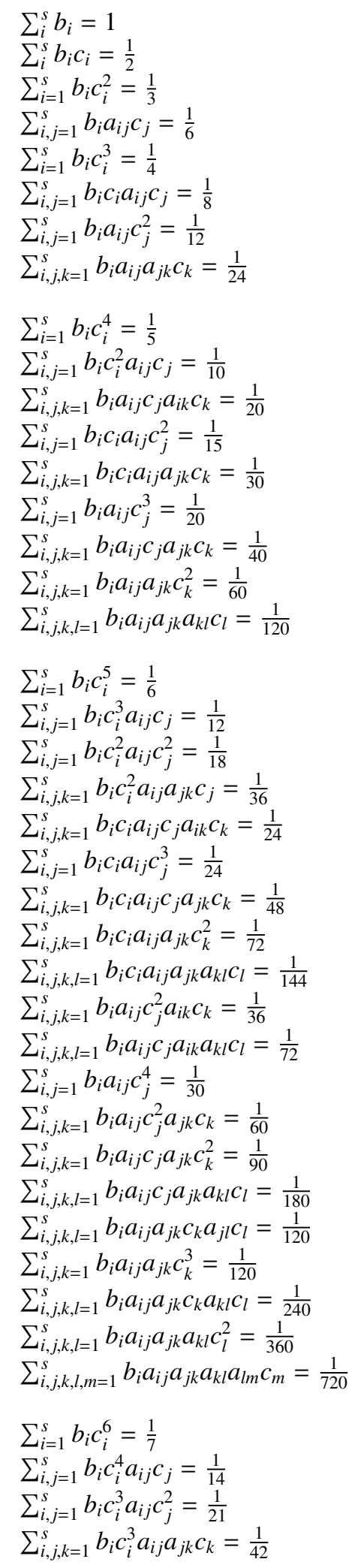

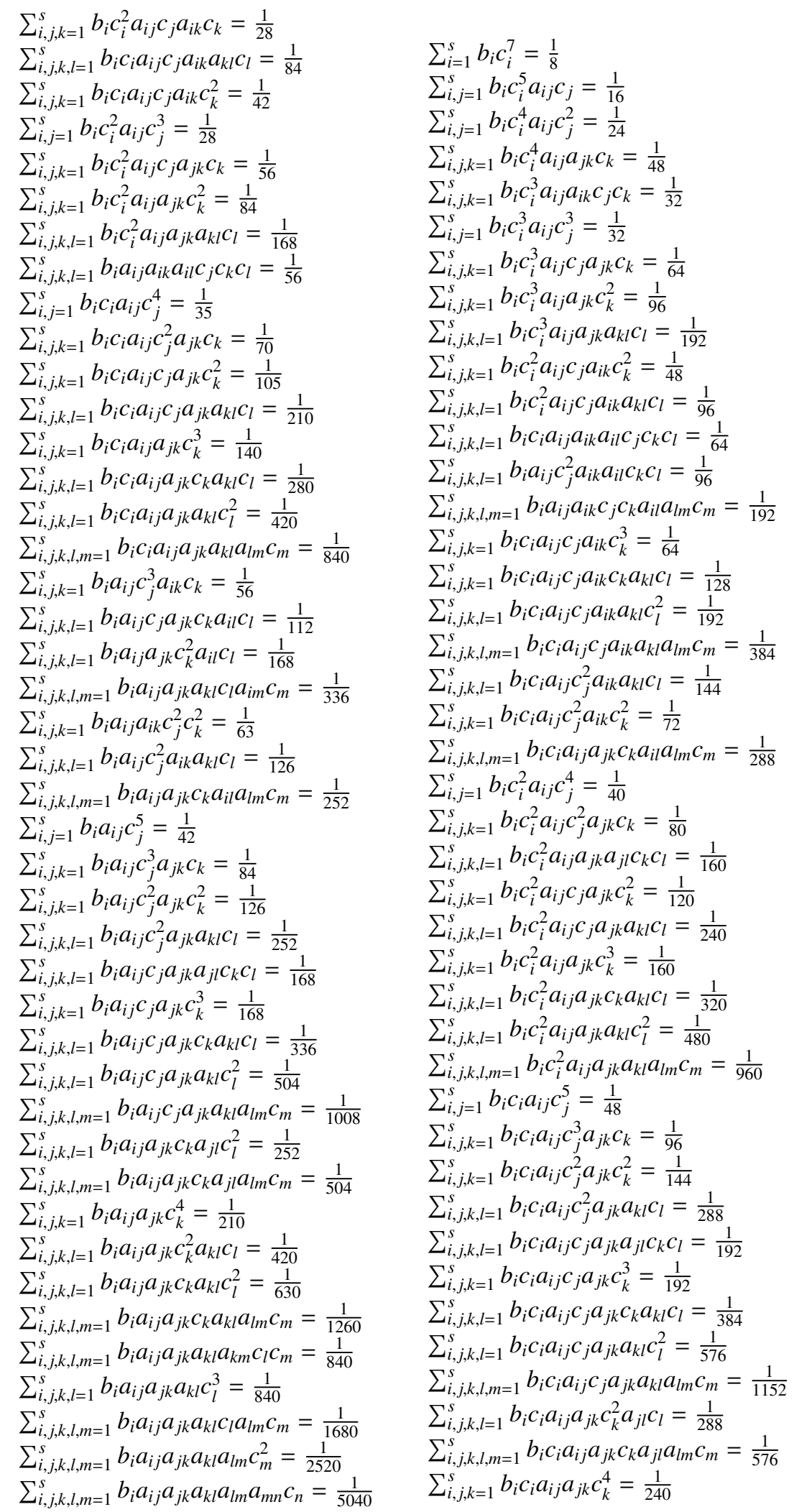

$\sum_{i, j, k=1}^{s} b_{i} c_{i}^{2} a_{i j} c_{j} a_{i k} c_{k}=\frac{1}{28}$

$\sum_{i, j, k, l=1}^{s} b_{i} c_{i} a_{i j} c_{j} a_{i k} a_{k l} c_{l}=\frac{1}{84}$

$\sum_{i, j, k=1}^{s} b_{i} c_{i} a_{i j} c_{j} a_{i k} c_{k}^{2}=\frac{1}{42}$

$\sum_{i, j=1}^{s} b_{i} c_{i}^{2} a_{i j} c_{j}^{3}=\frac{1}{28}$

$\sum_{i, j, k=1}^{s} b_{i} c_{i}^{2} a_{i j} c_{j} a_{j k} c_{k}=\frac{1}{56}$

$\sum_{i, j, k=1}^{s} b_{i} c_{i}^{2} a_{i j} a_{j k} c_{k}^{2}=\frac{1}{84}$

$\sum_{i, j, k, l=1}^{s} b_{i} c_{i}^{2} a_{i j} a_{j k} a_{k l} c_{l}=\frac{1}{168}$

$\sum_{i, j, k, l=1}^{s} b_{i} a_{i j} a_{i k} a_{i l} c_{j} c_{k} c_{l}=\frac{1}{56}$

$\sum_{i, j=1}^{s} b_{i} c_{i} a_{i j} c_{j}^{4}=\frac{1}{35}$

$\sum_{i, j, k=1}^{s} b_{i} c_{i} a_{i j} c_{j}^{2} a_{j k} c_{k}=\frac{1}{70}$

$\sum_{i, j, k=1}^{s} b_{i} c_{i} a_{i j} c_{j} a_{j k} c_{k}^{2}=\frac{1}{105}$

$\sum_{i, j, k, l=1}^{s} b_{i} c_{i} a_{i j} c_{j} a_{j k} a_{k l} c_{l}=\frac{1}{210}$

$\sum_{i, j, k=1}^{s} b_{i} c_{i} a_{i j} a_{j k} c_{k}^{3}=\frac{1}{140}$

$\sum_{i, j, k, l=1}^{s} b_{i} c_{i} a_{i j} a_{j k} c_{k} a_{k l} c_{l}=\frac{1}{280}$

$\sum_{i, j, k, l=1}^{s} b_{i} c_{i} a_{i j} a_{j k} a_{k l} c_{l}^{2}=\frac{1}{420}$

$\sum_{i, j, k, l, m=1}^{s} b_{i} c_{i} a_{i j} a_{j k} a_{k l} a_{l m} c_{m}=\frac{1}{840}$

$\sum_{i, j, k=1}^{s} b_{i} a_{i j} c_{j}^{3} a_{i k} c_{k}=\frac{1}{56}$

$\sum_{i, j, k, l=1}^{s} b_{i} a_{i j} c_{j} a_{j k} c_{k} a_{i l} c_{l}=\frac{1}{112}$

$\sum_{i, j, k, l=1}^{s} b_{i} a_{i j} a_{j k} c_{k}^{2} a_{i l} c_{l}=\frac{1}{168}$

$\sum_{i, j, k, l, m=1}^{s} b_{i} a_{i j} a_{j k} a_{k l} c_{l} a_{i m} c_{m}=\frac{1}{336}$

$\sum_{i, j, k=1}^{s} b_{i} a_{i j} a_{i k} c_{j}^{2} c_{k}^{2}=\frac{1}{63}$

$\sum_{i, j, k, l=1}^{s} b_{i} a_{i j} c_{j}^{2} a_{i k} a_{k l} c_{l}=\frac{1}{126}$

$\sum_{i, j, k, l, m=1}^{s} b_{i} a_{i j} a_{j k} c_{k} a_{i l} a_{l m} c_{m}=\frac{1}{252}$

$\sum_{i, j=1}^{s} b_{i} a_{i j} c_{j}^{5}=\frac{1}{42}$

$\sum_{i, j, k=1}^{s} b_{i} a_{i j} c_{j}^{3} a_{j k} c_{k}=\frac{1}{84}$

$\sum_{i, j, k=1}^{s} b_{i} a_{i j} c_{j}^{2} a_{j k} c_{k}^{2}=\frac{1}{126}$

$\sum_{i, j, k, l=1}^{s} b_{i} a_{i j} c_{j}^{2} a_{j k} a_{k l} c_{l}=\frac{1}{252}$

$\sum_{i, j, k, l=1}^{s} b_{i} a_{i j} c_{j} a_{j k} a_{j l} c_{k} c_{l}=\frac{1}{168}$

$\sum_{i, j, k=1}^{s} b_{i} a_{i j} c_{j} a_{j k} c_{k}^{3}=\frac{1}{168}$

$\sum_{i, j, k, l=1}^{s} b_{i} a_{i j} c_{j} a_{j k} c_{k} a_{k l} c_{l}=\frac{1}{336}$

$\sum_{i, j, k, l=1}^{s} b_{i} a_{i j} c_{j} a_{j k} a_{k l} c_{l}^{2}=\frac{1}{504}$

$\sum_{i, j, k, l, m=1}^{s} b_{i} a_{i j} c_{j} a_{j k} a_{k l} a_{l m} c_{m}=\frac{1}{1008}$

$\sum_{i, j, k, l=1}^{s} b_{i} a_{i j} a_{j k} c_{k} a_{j l} c_{l}^{2}=\frac{1}{252}$

$\sum_{i, j, k, l, m=1}^{s,} b_{i} a_{i j} a_{j k} c_{k} a_{j l} a_{l m} c_{m}=\frac{1}{504}$

$\sum_{i, j, k=1}^{s} b_{i} a_{i j} a_{j k} c_{k}^{4}=\frac{1}{210}$

$\sum_{i, j, k, l=1}^{s} b_{i} a_{i j} a_{j k} c_{k}^{2} a_{k l} c_{l}=\frac{1}{420}$

$\sum_{i, j, k, l=1}^{s} b_{i} a_{i j} a_{j k} c_{k} a_{k l} c_{l}^{2}=\frac{1}{630}$

$\sum_{i, j, k, l, m=1}^{s} b_{i} a_{i j} a_{j k} c_{k} a_{k l} a_{l m} c_{m}=\frac{1}{1260}$

$\sum_{i, j, k, l, m=1}^{s} b_{i} a_{i j} a_{j k} a_{k l} a_{k m} c_{l} c_{m}=\frac{1}{840}$

$\sum_{i, j, k, l=1}^{s} b_{i} a_{i j} a_{j k} a_{k l} c_{l}^{3}=\frac{1}{840}$

$\sum_{i, j, k, l, m=1}^{S} b_{i} a_{i j} a_{j k} a_{k l} c_{l} a_{l m} c_{m}=\frac{1}{1680}$

\begin{tabular}{l}
$\sum_{i, j, k, l, m=1}^{s} b_{i} a_{i j} a_{j k} a_{k l} a_{l m} a_{m n} c_{n}=\frac{1}{5040}$ \\
\hline
\end{tabular}

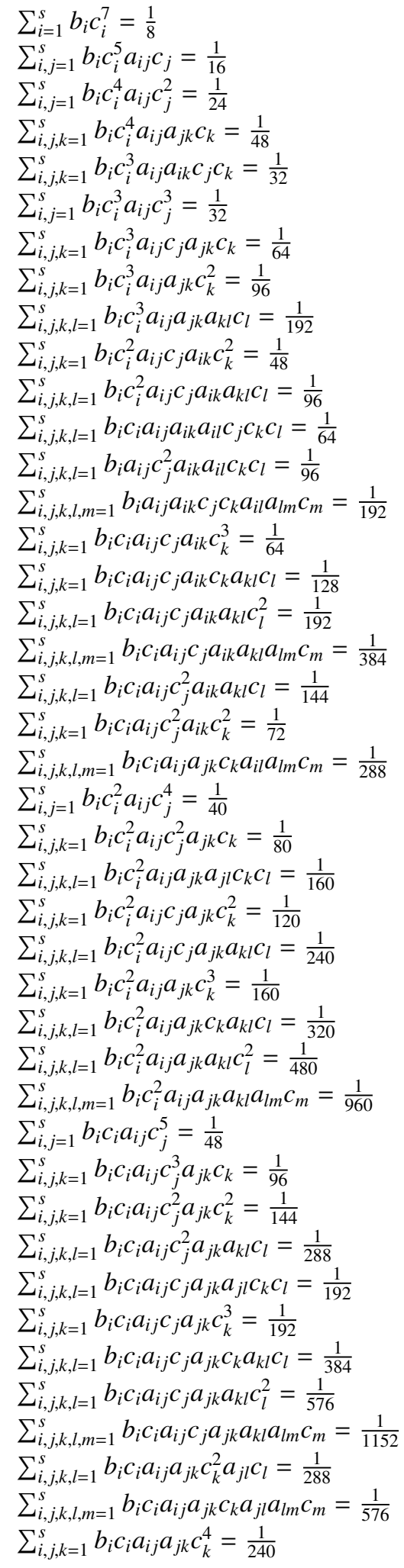




\begin{tabular}{|c|c|}
\hline 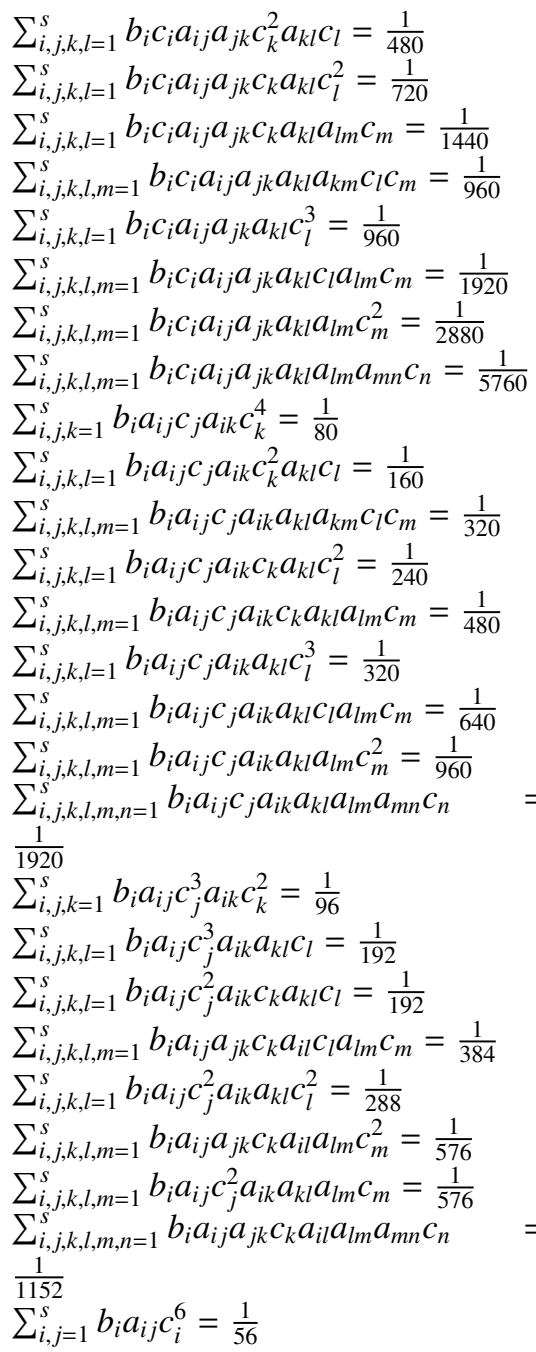 & $\begin{array}{l}\sum_{i, j, k=1}^{s} b_{i} a_{i j} c_{j}^{4} a_{j k} c_{k}=\frac{1}{112} \\
\sum_{i, j, k=1}^{s} b_{i} a_{i j} c_{j}^{3} a_{j k} c_{k}^{2}=\frac{1}{168} \\
\sum_{i, j, k, l=1}^{s} b_{i} a_{i j} c_{j}^{3} a_{j k} a_{k l} c_{l}=\frac{1}{336} \\
\sum_{i, j, k, l=1}^{s} b_{i} a_{i j} c_{j}^{2} a_{j k} c_{l} a_{j l} c_{k}=\frac{1}{224} \\
\sum_{i, j, k, l, m=1}^{s} b_{i} a_{i j} c_{j} a_{j k} c_{k} a_{j l} a_{l m} c_{m}=\frac{1}{672} \\
\sum_{i, j, k, l=1}^{s} b_{i} a_{i j} c_{j} a_{j k} c_{k} a_{j l} c_{l}^{2}=\frac{1}{336} \\
\sum_{i, j, k=1}^{s} b_{i} a_{i j} c_{j}^{2} a_{j k} c_{k}^{3}=\frac{1}{224} \\
\sum_{i, j, k, l=1}^{s} b_{i} a_{i j} c_{j}^{2} a_{j k} c_{k} a_{k l} c_{l}=\frac{1}{448} \\
\sum_{i, j, k, l=1}^{s} b_{i} a_{i j} c_{j}^{2} a_{j k} a_{k l} c_{l}^{2}=\frac{1}{672} \\
\sum_{i, j, k, l, m=1}^{s} b_{i} a_{i j} c_{j}^{2} a_{j k} a_{k l} a_{l m} c_{m}=\frac{1}{1344} \\
\sum_{i, j, k, l, m=1}^{s} b_{i} a_{i j} a_{j k} a_{j l} a_{j m} c_{k} c_{l} c_{m}=\frac{1}{448} \\
\sum_{i, j, k=1}^{s} b_{i} a_{i j} c_{j} a_{j k} c_{k}^{4}=\frac{1}{280} \\
\sum_{i, j, k, l=1}^{s} b_{i} a_{i j} c_{j} a_{j k} c_{k}^{2} a_{k l} c_{l}=\frac{1}{560} \\
\sum_{i, j, k, l=1}^{s} b_{i} a_{i j} c_{j} a_{j k} a_{k l} a_{k m} c_{m} c_{l}=\frac{1}{1120} \\
\sum_{i, j, k, l=1}^{s} b_{i} a_{i j} c_{j} a_{j k} c_{k} a_{k l} c_{l}^{2}=\frac{1}{840} \\
\sum_{i, j, k, l, m=1}^{s} b_{i} a_{i j} c_{j} a_{j k} c_{k} a_{k l} a_{l m} c_{m}=\frac{1}{1680} \\
\sum_{i, j, k=1}^{s} b_{i} a_{i j} c_{j} a_{j k} a_{k l} c_{l}^{3}=\frac{1}{1120} \\
\sum_{i, j, k, l, m=1}^{s} b_{i} a_{i j} c_{j} a_{j k} a_{k l} c_{l} a_{l m} c_{m}=\frac{1}{2240} \\
\sum_{i, j, k, l, m=1}^{s} b_{i} a_{i j} c_{j} a_{j k} a_{k l} a_{l m} c_{m}^{2}=\frac{1}{3360} \\
\sum_{i, j, k, l, m, n=1}^{s} b_{i} a_{i j} c_{j} a_{j k} a_{k l} a_{l m} a_{m n} c_{n} \\
\frac{1}{6720} \\
\sum_{i, j, k, l=1}^{s} b_{i} a_{i j} a_{j k} c_{k} a_{j l} c_{l}^{3}=\frac{1}{448} \\
\sum_{i, j, k, l, m=1}^{s} b_{i} a_{i j} a_{j k} c_{k} a_{j l} c_{l} a_{l m} c_{m}=\frac{1}{896} \\
\sum_{i, j, k, l, m=1}^{s} b_{i} a_{i j} a_{j k} c_{k} a_{j l} a_{l m} c_{m}^{2}=\frac{1}{1344} \\
\sum_{i, j, k, l, m, n=1}^{s} b_{i} a_{i j} a_{j k} c_{k} a_{j l} a_{l m} a_{m n} c_{n} \\
\frac{1}{2688} \\
\sum_{i, j, k, l=1}^{s} b_{i} a_{i j} a_{j k} c_{k}^{2} a_{j l} c_{l}^{2}=\frac{1}{500} \\
=1\end{array}$ \\
\hline
\end{tabular}

$\sum_{i, j, k, l, m=1}^{s} b_{i} a_{i j} a_{j k} c_{k}^{2} a_{j l} a_{l m} c_{m}=\frac{1}{1008}$ $\sum_{i, j, k, l, m, n=1}^{s} b_{i} a_{i j} a_{j k} a_{k l} c_{l} a_{j m} a_{m n} c_{n}=$ $\frac{1}{2016}$ $\sum_{i, j, k=1}^{s} b_{i} a_{i j} a_{j k} c_{k}^{5}=\frac{1}{336}$ $\sum_{i, j, k, l=1}^{S} b_{i} a_{i j} a_{j k} c_{k}^{3} a_{k l} c_{l}=\frac{1}{672}$ $\sum_{i, j, k, l=1}^{s} b_{i} a_{i j} a_{j k} c_{k}^{2} a_{k l} c_{l}^{2}=\frac{1}{1008}$ $\sum_{i, j, k, l, m=1}^{s} b_{i} a_{i j} a_{j k} c_{k}^{2} a_{k l} a_{l m} c_{m}=\frac{1}{2016}$ $\sum_{i, j, k, l, m=1}^{s} b_{i} a_{i j} a_{j k} c_{k} a_{k l} a_{k m} c_{m} c_{l}=\frac{1}{1344}$ $\sum_{i, j, k, l=1}^{s} b_{i} a_{i j} a_{j k} c_{k} a_{k l} c_{l}^{3}=\frac{1}{1344}$ $\sum_{i, j, k, l, m=1}^{s} b_{i} a_{i j} a_{j k} c_{k} a_{k l} c_{l} a_{l m} c_{m}=\frac{1}{2688}$ $\sum_{i, j, k, l, m=1}^{s} b_{i} a_{i j} a_{j k} c_{k} a_{k l} a_{l m} c_{m}^{2}=\frac{1}{4032}$ $\sum_{i, j, k, l, m, n=1}^{s} b_{i} a_{i j} a_{j k} c_{k} a_{k l} a_{l m} a_{m n} c_{n}=$ $\frac{1}{8064}$ $\sum_{i, j, k, l, m=1}^{s} b_{i} a_{i j} a_{j k} a_{k l} c_{l}^{2} a_{k m} c_{m}=\frac{1}{2016}$ $\sum_{i, j, k, l, m, n=1}^{s} b_{i} a_{i j} a_{j k} a_{k l} c_{l} a_{k m} a_{m n} c_{m}=$ $\frac{1}{4032}$ $\sum_{i, j, k, l=1}^{s} b_{i} a_{i j} a_{j k} a_{k l} c_{l}^{4}=\frac{1}{1680}$ $\sum_{i, j, k, l, m=1}^{s} b_{i} a_{i j} a_{j k} a_{k l} c_{l}^{2} a_{l m} c_{m}=\frac{1}{3360}$ $\sum_{i, j, k, l, m=1}^{s} b_{i} a_{i j} a_{j k} a_{k l} a_{l m} c_{l} c_{m}^{2}=\frac{1}{5040}$ $\sum_{i, j, k, l, m, n=1}^{s} b_{i} a_{i j} a_{j k} a_{k l} a_{l m} a_{m n} c_{n} c_{l}=$ $\frac{1}{10080}$ $\sum_{i, j, k, l, m, n=1}^{s} b_{i} a_{i j} a_{j k} a_{k l} a_{l m} a_{l n} c_{n} c_{m} \quad=$ $\frac{1}{6720}$ $\sum_{i, j, k, l, m=1}^{s} b_{i} a_{i j} a_{j k} a_{k l} a_{l m} c_{m}^{3}=\frac{1}{6720}$ $\sum_{i, j, k, l, m, n=1}^{s} b_{i} a_{i j} a_{j k} a_{k l} a_{l m} a_{m n} c_{n} c_{m}=$ $\frac{1}{13440}$ $\sum_{i, j, k, l, m, n=1}^{s} b_{i} a_{i j} a_{j k} a_{k l} a_{l m} a_{m n} c_{n}^{2}=\frac{1}{20160}$ $\sum_{i, j, k, l, m, n, o=1}^{s} b_{i} a_{i j} a_{j k} a_{k l} a_{l m} a_{m n} a_{n o} c_{o}=$ $\frac{1}{40320}$ 


\section{Appendix B}

\section{The successive derivatives of the exact solution}

$\alpha_{i}, \beta_{j} \in \mathbb{N}^{*}$

- $\dot{y}=f$

- $\ddot{y}=f^{\prime}(f)$

- $y^{(3)}=f^{\prime \prime}(f, f)+f^{\prime}\left(f^{\prime}(f)\right)$

- $y^{(4)}=f^{(3)}(f, f, f)+3 f^{\prime \prime}\left(f^{\prime}(f), f\right)+f^{\prime}\left(f^{\prime \prime}(f, f)\right)+f^{\prime}\left(f^{\prime}\left(f^{\prime}(f)\right)\right)$

- $y^{(5)}=f^{(4)}(f, f, f, f)+6 f^{(3)}\left(f^{\prime}(f), f, f\right)+4 f^{\prime \prime}\left(f^{\prime \prime}(f, f), f\right)+$ $4 f^{\prime \prime}\left(f^{\prime}\left(f^{\prime}(f)\right), f\right)+3 f^{\prime \prime}\left(f^{\prime}(f), f^{\prime}(f)\right)+f^{\prime}\left(f^{(3)}(f, f, f)+\right.$ $3 f^{\prime}\left(f^{\prime \prime}\left(f^{\prime} f, f\right)\right)+f^{\prime}\left(f^{\prime}\left(f^{\prime \prime}(f, f)\right)\right)+f^{\prime}\left(f^{\prime}\left(f^{\prime}\left(f^{\prime}(f)\right)\right)\right)$

- $y^{(6)}=f^{(5)}(f, f, f, f, f)+10 f^{(4)}\left(f, f, f, f^{\prime} f\right)+10 f^{(3)}\left(f, f, f^{\prime \prime}(f, f)\right)+$ $10 f^{(3)}\left(f, f, f^{\prime}\left(f^{\prime}(f)\right)\right)+15 f^{(3)}\left(f, f^{\prime} f, f^{\prime} f\right)+5 f^{\prime \prime}\left(f, f^{(3)}(f, f, f)\right)+$ $15 f^{\prime \prime}\left(f, f^{\prime \prime}\left(f^{\prime}(f), f\right)\right)+5 f^{\prime \prime}\left(f, f^{\prime}\left(f^{\prime \prime}(f, f)\right)\right)+5 f^{\prime \prime}\left(f, f^{\prime}\left(f^{\prime}\left(f^{\prime}(f)\right)\right)\right)+$ $10 f^{\prime \prime}\left(f^{\prime} f, f^{\prime \prime}(f, f)\right)+10 f^{\prime \prime}\left(f^{\prime} f, f^{\prime}\left(f^{\prime}(f)\right)\right)+f^{\prime} f^{(4)}(f, f, f, f)+$ $6 f^{\prime} f^{(3)}\left(f^{\prime}(f), f, f\right)+4 f^{\prime} f^{\prime \prime}\left(f^{\prime \prime}(f, f), f\right)+4 f^{\prime} f^{\prime \prime}\left(f^{\prime}\left(f^{\prime}(f)\right), f\right)+$ $3 f^{\prime} f^{\prime \prime}\left(f^{\prime}(f), f^{\prime}(f)\right)+f^{\prime} f^{\prime}\left(f^{(3)}(f, f, f)+3 f^{\prime} f^{\prime}\left(f^{\prime \prime}\left(f^{\prime} f, f\right)\right)+\right.$ $f^{\prime} f^{\prime}\left(f^{\prime}\left(f^{\prime \prime}(f, f)\right)\right)+f^{\prime} f^{\prime}\left(f^{\prime}\left(f^{\prime}\left(f^{\prime}(f)\right)\right)\right)$

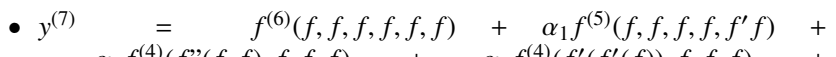
$\alpha_{2} f^{(4)}\left(f^{\prime \prime}(f, f), f, f, f\right)+\alpha_{3} f^{(4)}\left(f^{\prime}\left(f^{\prime}(f)\right), f, f, f\right)+$ $\alpha_{4} f^{(4)}\left(f^{\prime} f, f^{\prime} f, f, f\right)+\alpha_{5} f^{(3)}\left(f, f^{\prime} f, f^{\prime \prime}(f, f)\right)+\alpha_{6} f^{(3)}\left(f, f^{\prime} f, f^{\prime}\left(f^{\prime}(f)\right)\right)+$ $\alpha_{7} f^{(3)}\left(f, f, f^{(3)}(f, f, f)\right)+\alpha_{8} f^{(3)}\left(f, f, f^{\prime \prime}\left(f^{\prime}(f), f\right)\right)+$ $\alpha_{9} f^{(3)}\left(f, f, f^{\prime}\left(f^{\prime \prime}(f, f)\right)\right)+\alpha_{10} f^{(3)}\left(f, f, f^{\prime}\left(f^{\prime}\left(f^{\prime}(f)\right)\right)\right)+$ $\alpha_{11} f^{(3)}\left(f^{\prime} f, f^{\prime} f, f^{\prime} f\right)+\alpha_{12} f^{\prime \prime}\left(f, f^{(4)}(f, f, f, f)\right)+\alpha_{13} f^{\prime \prime}\left(f, f^{(3)}\left(f^{\prime}(f), f, f\right)\right)+$ $\alpha_{14} f^{\prime \prime}\left(f, f^{\prime \prime}\left(f f^{\prime \prime}(f, f), f\right)\right)+\alpha_{15} f^{\prime \prime}\left(f, f^{\prime \prime}\left(f^{\prime}\left(f^{\prime}(f)\right)\right)+\right.$ $\alpha_{17} f^{\prime \prime}\left(f, f^{\prime \prime}\left(f^{\prime}(f), f^{\prime}(f)\right)\right)+\alpha_{18} f^{\prime \prime}\left(f, f^{\prime}\left(f^{(3)}(f, f, f)\right)+\right.$ $\alpha_{19} f^{\prime \prime}\left(f, f^{\prime}\left(f^{\prime \prime}\left(f^{\prime} f, f\right)\right)\right)+\alpha_{20} f^{\prime \prime}\left(f, f^{\prime}\left(f^{\prime}\left(f^{\prime \prime}(f, f)\right)\right)\right)+$ $\alpha_{21} f^{\prime \prime}\left(f, f^{\prime}\left(f^{\prime}\left(f^{\prime}\left(f^{\prime}(f)\right)\right)\right)\right)+\alpha_{22} f^{\prime \prime}\left(f^{\prime} f, f^{(3)}(f, f, f)\right)+$ $\alpha_{23} f^{\prime \prime}\left(f^{\prime} f, f^{\prime \prime}\left(f^{\prime}(f), f\right)\right)+\alpha_{24} f^{\prime \prime}\left(f^{\prime} f, f^{\prime}\left(f f^{\prime \prime}(f, f)\right)\right)+$ $\alpha_{25} f^{\prime \prime}\left(f^{\prime} f, f^{\prime}\left(f^{\prime}\left(f^{\prime}(f)\right)\right)\right)+\alpha_{26} f^{\prime \prime}\left(f^{\prime \prime}(f, f), f^{\prime \prime}(f, f)\right)+$ $\alpha_{27} f^{\prime \prime}\left(f^{\prime \prime}(f, f), f^{\prime}\left(f^{\prime}(f)\right)\right)+\alpha_{28} f^{\prime \prime}\left(f^{\prime}\left(f^{\prime}(f)\right), f^{\prime}\left(f^{\prime}(f)\right)\right)+$ $f^{\prime} f^{(5)}(f, f, f, f, f)+\alpha_{29} f^{\prime} f^{(4)}\left(f, f, f, f^{\prime} f\right)+\alpha_{30} f^{\prime} f^{(3)}\left(f, f, f^{\prime \prime}(f, f)\right)+$ $\alpha_{31} f^{\prime} f^{(3)}\left(f, f, f^{\prime}\left(f^{\prime}(f)\right)\right)+\alpha_{32} f^{\prime} f^{(3)}\left(f, f^{\prime} f, f^{\prime} f\right)+$ $\alpha_{33} f^{\prime} f^{\prime \prime}\left(f, f^{(3)}(f, f, f)\right)+\alpha_{34} f^{\prime} f^{\prime \prime}\left(f, f^{\prime \prime}\left(f^{\prime}(f), f\right)\right)+$ $\alpha_{35} f^{\prime} f^{\prime \prime}\left(f, f^{\prime}\left(f^{\prime \prime}(f, f)\right)\right)+\alpha_{36} f^{\prime} f^{\prime \prime}\left(f, f^{\prime}\left(f^{\prime}\left(f^{\prime}(f)\right)\right)\right)+$ $\alpha_{37} f^{\prime} f^{\prime \prime}\left(f^{\prime} f, f^{\prime \prime}(f, f)\right)+\alpha_{38} f^{\prime} f^{\prime \prime}\left(f^{\prime} f, f^{\prime}\left(f^{\prime}(f)\right)\right)+f^{\prime} f^{\prime} f^{(4)}(f, f, f, f)+$ $\alpha_{39} f^{\prime} f^{\prime} f^{(3)}\left(f^{\prime}(f), f, f\right)+\alpha_{40} f^{\prime} f^{\prime} f^{\prime \prime}\left(f^{\prime \prime}(f, f), f\right)+\alpha_{41} f^{\prime} f^{\prime} f^{\prime \prime}\left(f^{\prime}\left(f^{\prime}(f)\right), f\right)+$ $\alpha_{42} f^{\prime} f^{\prime} f^{\prime \prime}\left(f^{\prime}(f), f^{\prime}(f)\right)+f^{\prime} f^{\prime} f^{\prime}\left(f^{(3)}(f, f, f)+\alpha_{43} f^{\prime} f^{\prime} f^{\prime}\left(f^{\prime \prime}\left(f^{\prime} f, f\right)\right)+\right.$ $f^{\prime} f^{\prime} f^{\prime}\left(f^{\prime}\left(f^{\prime \prime}(f, f)\right)\right)+f^{\prime} f^{\prime} f^{\prime}\left(f^{\prime}\left(f^{\prime}\left(f^{\prime}(f)\right)\right)\right)$

- $y^{(8)}=f^{(7)}(f, f, f, f, f, f, f)+\beta_{1} f^{(6)}\left(f, f, f, f, f, f^{\prime} f\right)+$ $\beta_{2} f^{(5)}\left(f, f, f, f, f^{\prime \prime}(f, f)\right)+\beta_{3} f^{(5)}\left(f, f, f, f, f^{\prime}\left(f^{\prime}(f)\right)\right)$ $+\beta_{4} f^{(5)}\left(f^{\prime} f, f^{\prime} f, f, f, f\right)+\beta_{5} f^{(4)}\left(f^{(3)}(f, f, f), f, f, f\right)+$ $\beta_{6} f^{(4)}\left(f^{\prime \prime}\left(f^{\prime}(f), f\right), f, f, f\right)+\beta_{7} f^{(4)}\left(f^{\prime}\left(f^{\prime \prime}(f, f)\right), f, f, f\right)$ $+\beta_{8} f^{(4)}\left(f^{\prime}\left(f^{\prime}\left(f^{\prime}(f)\right)\right), f, f, f\right)+\beta_{9} f^{(4)}\left(f, f, f^{\prime} f, f^{\prime \prime}(f, f)\right)+$ $\beta_{10} f^{(4)}\left(f, f, f^{\prime} f, f^{\prime}\left(f^{\prime}(f)\right)\right)+\beta_{11} f^{(4)}\left(f, f^{\prime} f, f^{\prime} f, f^{\prime} f\right)$ $+\beta_{12} f^{(3)}\left(f^{(3)}(f, f, f), f, f^{\prime} f\right)+\beta_{13} f^{(3)}\left(f^{\prime \prime}\left(f^{\prime}(f), f\right), f, f^{\prime} f\right)+$ $\beta_{14} f^{(3)}\left(f^{\prime}\left(f^{\prime \prime}(f, f)\right), f, f^{\prime} f\right)+\beta_{15} f^{(3)}\left(f^{\prime}\left(f^{\prime}\left(f^{\prime}(f)\right)\right), f, f^{\prime} f\right)$

$+\beta_{16} f^{(3)}\left(f^{\prime \prime}(f, f), f^{\prime} f, f^{\prime} f\right)+\beta_{17} f^{(3)}\left(f^{\prime}\left(f^{\prime}(f)\right), f^{\prime} f, f^{\prime} f\right)+$ $\beta_{18} f^{(3)}\left(f^{\prime \prime}(f, f), f^{\prime \prime}(f, f), f\right)+\beta_{19} f^{(3)}\left(f^{\prime \prime}(f, f), f^{\prime}\left(f^{\prime}(f)\right), f\right)$ $+\beta_{20} f^{(3)}\left(f^{\prime}\left(f^{\prime}(f)\right), f^{\prime}\left(f^{\prime}(f)\right), f\right)+\beta_{21} f^{(3)}\left(f, f, f^{(4)}(f, f, f, f)\right)+$ $\beta_{22} f^{(3)}\left(f, f, f^{(3)}\left(f^{\prime}(f), f, f\right)\right)+\beta_{23} f^{(3)}\left(f, f, f^{\prime \prime}\left(f^{\prime \prime}(f, f), f\right)\right)$ $+\beta_{24} f^{(3)}\left(f, f, f^{\prime \prime}\left(f^{\prime}\left(f^{\prime}(f)\right), f\right)\right)+\beta_{25} f^{(3)}\left(f, f, f^{\prime \prime}\left(f^{\prime}(f), f^{\prime}(f)\right)\right)+$ $\beta_{26} f^{(3)}\left(f, f, f^{\prime}\left(f^{(3)}(f, f, f)\right)+\beta_{27} f^{(3)}\left(f, f, f^{\prime}\left(f^{\prime \prime}\left(f^{\prime} f, f\right)\right)\right)\right.$ $+21 f^{(3)}\left(f, f, f^{\prime}\left(f^{\prime}\left(f^{\prime \prime}(f, f)\right)\right)\right)+\beta_{28} f^{(3)}\left(f, f, f^{\prime}\left(f^{\prime}\left(f^{\prime}\left(f^{\prime}(f)\right)\right)\right)\right)+$ $\beta_{29} f^{\prime \prime}\left(f^{(5)}(f, f, f, f, f), f\right)+\beta_{30} f^{\prime \prime}\left(10 f^{(4)}\left(f, f, f, f^{\prime} f\right), f\right)$ $+\beta_{31} f^{\prime \prime}\left(10 f^{(3)}\left(f, f, f^{\prime \prime}(f, f)\right), f\right)+\beta_{32} f^{\prime \prime}\left(f^{(3)}\left(f, f, f^{\prime}\left(f^{\prime}(f)\right)\right), f\right)+$ $\beta_{33} f^{\prime \prime}\left(f^{(3)}\left(f, f^{\prime} f, f^{\prime} f\right), f\right)+\beta_{34} f^{\prime \prime}\left(f^{\prime \prime}\left(f, f^{(3)}(f, f, f)\right), f\right)$ $+\beta_{35} f^{\prime \prime}\left(f^{\prime \prime}\left(f, f^{\prime \prime}\left(f^{\prime}(f), f\right)\right), f\right)+f^{\prime \prime}\left(5 f^{\prime \prime}\left(f, f^{\prime}\left(f^{\prime \prime}(f, f)\right)\right), f\right)+$ $f^{\prime \prime}\left(5 f^{\prime \prime}\left(f, f^{\prime}\left(f^{\prime}\left(f^{\prime}(f)\right)\right)\right), f\right)+\beta_{36} f^{\prime \prime}\left(f^{\prime \prime}\left(f^{\prime} f, f^{\prime \prime}(f, f)\right), f\right)$ $+\beta_{37} f^{\prime \prime}\left(f^{\prime \prime}\left(f^{\prime} f, f^{\prime}\left(f^{\prime}(f)\right)\right), f\right)+\beta_{38} f^{\prime \prime}\left(f^{\prime} f^{(4)}(f, f, f, f), f\right)+$ $\beta_{39} f^{\prime \prime}\left(f^{\prime} f^{(3)}\left(f^{\prime}(f), f, f\right), f\right)$

$+\beta_{40} f^{\prime \prime}\left(f^{\prime} f^{\prime \prime}\left(f^{\prime \prime}(f, f), f\right), f\right)+\beta_{41} f^{\prime \prime}\left(f^{\prime} f^{\prime \prime}\left(f^{\prime}\left(f^{\prime}(f)\right), f\right), f\right)+$ $\beta_{42} f^{\prime \prime}\left(f^{\prime} f^{\prime \prime}\left(f^{\prime}(f), f^{\prime}(f)\right), f\right)$

$+\beta_{43} f^{\prime \prime}\left(f^{\prime} f^{\prime}\left(f^{(3)}(f, f, f), f\right)+\beta_{44} f^{\prime \prime}\left(f^{\prime} f^{\prime}\left(f^{\prime \prime}\left(f^{\prime} f, f\right)\right), f\right)+\right.$ $\beta_{45} f^{\prime \prime}\left(f^{\prime} f^{\prime}\left(f^{\prime}\left(f^{\prime \prime}(f, f)\right)\right), f\right)+\beta_{46} f^{\prime \prime}\left(f^{\prime} f^{\prime}\left(f^{\prime}\left(f^{\prime}\left(f^{\prime}(f)\right)\right)\right), f\right)$ $+\beta_{47} f^{\prime \prime}\left(f^{\prime} f, f^{(4)}(f, f, f, f)\right)+\beta_{48} f^{\prime \prime}\left(f^{\prime} f, f^{(3)}\left(f^{\prime}(f), f, f\right)\right)+$ $\beta_{49} f^{\prime \prime}\left(f^{\prime} f, f^{\prime \prime}\left(f^{\prime \prime}(f, f), f\right)\right)+\beta_{50} f^{\prime \prime}\left(f^{\prime} f, f^{\prime \prime}\left(f^{\prime}\left(f^{\prime}(f)\right), f\right)\right)$

$+\beta_{51} f^{\prime \prime}\left(f^{\prime} f, f^{\prime \prime}\left(f^{\prime}(f), f^{\prime}(f)\right)\right)+\beta_{52} f^{\prime \prime}\left(f^{\prime} f, f^{\prime}\left(f^{(3)}(f, f, f)\right)+\right.$ $\beta_{53} f^{\prime \prime}\left(f^{\prime} f, f^{\prime}\left(f^{\prime \prime}\left(f^{\prime} f, f\right)\right)\right)+\beta_{54} f^{\prime \prime}\left(f^{\prime} f, f^{\prime}\left(f^{\prime}\left(f^{\prime \prime}(f, f)\right)\right)\right)$

$+\beta_{55} f^{\prime \prime}\left(f^{\prime} f, f^{\prime}\left(f^{\prime}\left(f^{\prime}\left(f^{\prime}(f)\right)\right)\right)\right)+\beta_{56} f^{\prime \prime}\left(f^{(3)}(f, f, f), f^{\prime \prime}(f, f)\right)+$ $\beta_{57} f^{\prime \prime}\left(f^{(3)}(f, f, f), f^{\prime}\left(f^{\prime}(f)\right)\right)+\beta_{58} f^{\prime \prime}\left(f^{\prime \prime}\left(f^{\prime}(f), f\right), f^{\prime \prime}(f, f)\right)$ $+\beta_{60} f^{\prime \prime}\left(f^{\prime}\left(f^{\prime \prime}(f, f)\right), f^{\prime}\left(f^{\prime}(f)\right)\right)+\beta_{61} f^{\prime \prime}\left(f^{\prime}\left(f^{\prime}\left(f^{\prime}(f)\right)\right), f^{\prime}\left(f^{\prime}(f)\right)\right)+$ $\beta_{62} f^{\prime \prime}\left(f^{\prime \prime}\left(f^{\prime}(f), f\right), f^{\prime \prime}(f, f)\right)+\beta_{63} f^{\prime \prime}\left(f^{\prime}\left(f^{\prime \prime}(f, f)\right), f^{\prime}\left(f^{\prime}(f)\right)\right)$ $+\beta_{64} f^{\prime \prime}\left(f^{\prime}\left(f^{\prime}\left(f^{\prime}(f)\right)\right), f^{\prime \prime}(f, f)\right)+f^{\prime} f^{(6)}(f, f, f, f, f, f)+$ $\beta_{65} f^{\prime} f^{(5)}\left(f, f, f, f, f^{\prime} f\right)+\beta_{66} f^{\prime} f^{(4)}\left(f^{\prime \prime}(f, f), f, f, f\right)$ $+\beta_{67} f^{\prime} f^{(4)}\left(f^{\prime}\left(f^{\prime}(f)\right), f, f, f\right)+\beta_{68} f^{\prime} f^{(4)}\left(f^{\prime} f, f^{\prime} f, f, f\right)+$ $\beta_{69} f^{\prime} f^{(3)}\left(f, f^{\prime} f, f^{\prime \prime}(f, f)\right)+\beta_{70} f^{\prime} f^{(3)}\left(f, f^{\prime} f, f^{\prime}\left(f^{\prime}(f)\right)\right)$ $+\beta_{71} f^{\prime} f^{(3)}\left(f, f, f^{(3)}(f, f, f)\right)+\beta_{72} f^{\prime} f^{(3)}\left(f, f, f^{\prime \prime}\left(f^{\prime}(f), f\right)\right)+$ $\beta_{73} f^{\prime} f^{(3)}\left(f, f, f^{\prime}\left(f^{\prime \prime}(f, f)\right)\right)+\beta_{74} f^{\prime} f^{(3)}\left(f, f, f^{\prime}\left(f^{\prime}\left(f^{\prime}(f)\right)\right)\right)$ $+\beta_{75} f^{\prime} f^{(3)}\left(f^{\prime} f, f^{\prime} f, f^{\prime} f\right)+\beta_{76} f^{\prime} f^{\prime \prime}\left(f, f^{(4)}(f, f, f, f)\right)+$ $\beta_{77} f^{\prime} f^{\prime \prime}\left(f, f^{(3)}\left(f^{\prime}(f), f, f\right)\right)+\beta_{78} f^{\prime} f^{\prime \prime}\left(f, f^{\prime \prime}\left(f^{\prime \prime}(f, f), f\right)\right)$ $+\beta_{79} f^{\prime} f^{\prime \prime}\left(f, f^{\prime \prime}\left(f^{\prime}\left(f^{\prime}(f)\right)\right)+\beta_{80} f^{\prime} f^{\prime \prime}\left(f, f^{\prime \prime}\left(f^{\prime}(f), f^{\prime}(f)\right)\right)+\right.$ $\beta_{81} f^{\prime} f^{\prime \prime}\left(f, f^{\prime}\left(f^{(3)}(f, f, f)\right)+\beta_{82} f^{\prime} f^{\prime \prime}\left(f, f^{\prime}\left(f^{\prime \prime}\left(f^{\prime} f, f\right)\right)\right)\right.$ $+\beta_{83} f^{\prime} f^{\prime \prime}\left(f, f^{\prime}\left(f^{\prime}\left(f f^{\prime \prime}(f, f)\right)\right)\right)+\beta_{84} f^{\prime} f^{\prime \prime}\left(f, f^{\prime}\left(f^{\prime}\left(f^{\prime}\left(f^{\prime}(f)\right)\right)\right)\right)+$ $\beta_{85} f^{\prime} f^{\prime \prime}\left(f^{\prime} f, f^{(3)}(f, f, f)\right)+\beta_{86} f^{\prime} f^{\prime \prime}\left(f^{\prime} f, f^{\prime \prime}\left(f^{\prime}(f), f\right)\right)$ $+\beta_{87} f^{\prime} f^{\prime \prime}\left(f^{\prime} f, f^{\prime}\left(f^{\prime \prime}(f, f)\right)\right)+\beta_{88} f^{\prime} f^{\prime \prime}\left(f^{\prime} f, f^{\prime}\left(f^{\prime}\left(f^{\prime}(f)\right)\right)\right)+$ $\beta_{89} f^{\prime} f^{\prime \prime}\left(f " '(f, f), f^{\prime \prime}(f, f)\right)+\beta_{90} f^{\prime} f^{\prime \prime}\left(f^{\prime \prime}(f, f), f^{\prime}\left(f^{\prime}(f)\right)\right)$ $+\beta_{91} f^{\prime} f^{\prime \prime}\left(f^{\prime}\left(f^{\prime}(f)\right), f^{\prime}\left(f^{\prime}(f)\right)\right)+f^{\prime} f^{\prime} f^{(5)}(f, f, f, f, f)+$ $\beta_{92} f^{\prime} f^{\prime} f^{(4)}\left(f, f, f, f^{\prime} f\right)+\beta_{93} f^{\prime} f^{\prime} f^{(3)}\left(f, f, f^{\prime \prime}(f, f)\right)$ $+\beta_{94} f^{\prime} f^{\prime} f^{(3)}\left(f, f, f^{\prime}\left(f^{\prime}(f)\right)\right)+\beta_{95} f^{\prime} f^{\prime} f^{(3)}\left(f, f^{\prime} f, f^{\prime} f\right)+$ $\beta_{96} f^{\prime} f^{\prime} f^{\prime \prime}\left(f, f^{(3)}(f, f, f)\right)+\beta_{97} f^{\prime} f^{\prime} f^{\prime \prime}\left(f, f^{\prime \prime}\left(f^{\prime}(f), f\right)\right)$ $+5 f^{\prime} f^{\prime} f^{\prime \prime}\left(f, f^{\prime}\left(f^{\prime \prime}(f, f)\right)\right)+\beta_{98} f^{\prime} f^{\prime} f^{\prime \prime}\left(f, f^{\prime}\left(f^{\prime}\left(f^{\prime}(f)\right)\right)\right)+$ $\beta_{99} f^{\prime} f^{\prime} f^{\prime \prime}\left(f^{\prime} f, f^{\prime \prime}(f, f)\right)+\beta_{100} f^{\prime} f^{\prime} f^{\prime \prime}\left(f^{\prime} f, f^{\prime}\left(f^{\prime}(f)\right)\right)$ $+f^{\prime} f^{\prime} f^{\prime} f^{(4)}(f, f, f, f)+\beta_{101} f^{\prime} f^{\prime} f^{\prime} f^{(3)}\left(f^{\prime}(f), f, f\right) \quad+$ $\beta_{102} f^{\prime} f^{\prime} f^{\prime} f^{\prime \prime}\left(f^{\prime \prime}(f, f), f\right)+\beta_{103} f^{\prime} f^{\prime} f^{\prime} f^{\prime \prime}\left(f^{\prime}\left(f^{\prime}(f)\right), f\right)$ $+\beta_{104} f^{\prime} f^{\prime} f^{\prime} f^{\prime \prime}\left(f^{\prime}(f), f^{\prime}(f)\right)+f^{\prime} f^{\prime} f^{\prime} f^{\prime}\left(f^{(3)}(f, f, f)+\right.$ $\beta_{105} f^{\prime} f^{\prime} f^{\prime} f^{\prime}\left(f^{\prime \prime}\left(f^{\prime} f, f\right)\right)+f^{\prime} f^{\prime} f^{\prime} f^{\prime}\left(f^{\prime}\left(f^{\prime \prime}(f, f)\right)\right)+$ $f^{\prime} f^{\prime} f^{\prime} f^{\prime}\left(f^{\prime}\left(f^{\prime}\left(f^{\prime}(f)\right)\right)\right)$ 